\title{
A STUDY OF PROPERTIES AND APPLICATIONS OF GAMMA DISTRIBUTION
}

\author{
Eric U. ${ }^{1^{*}}$, Oti Michael O. Olusola ${ }^{2}$ and Francis C. Eze ${ }^{2}$ \\ ${ }^{1}$ Department of Statistics, Federal Polytechnic, Ekowe Bayelsa State Nigeria \\ ${ }^{2}$ Department of Statistics, Nnamdi Azikiwe University, Awka Anambra State Nigeria \\ *Corresponding Author: Eric U. Oti eluchcollections@gmail.com (+2348037979262)
}

\section{Cite this article:}

Eric U., Oti M.O.O., Francis C.E. (2021), A Study of Properties and Applications of Gamma Distribution. African Journal of Mathematics and Statistics Studies 4(2), 52-65. DOI: 10.52589/AJMSSMR0DQ1DG.

\section{Manuscript History}

Received: 28 May 2021

Accepted: 25 June 2021

Published: 8 July 2021

Copyright $\odot 2020$ The Author(s). This is an Open Access article distributed under the terms of Creative Commons AttributionNonCommercial-NoDerivatives 4.0 International (CC BY-NC-ND

4.0 ), which permits anyone to share, use, reproduce and redistribute in any medium, provided the original author and source are credited.
ABSTRACT: The gamma distribution is one of the continuous distributions; the distributions are very versatile and give useful presentations of many physical situations. They are perhaps the most applied statistical distribution in the area of reliability. In this paper, we present the study of properties and applications of gamma distribution to real life situations such as fitting the gamma distribution into data, burn-out time of electrical devices and reliability theory. The study employs the moment generating function approach and the special case of gamma distribution to show that the gamma distribution is a legitimate continuous probability distribution showing its characteristics.

KEYWORDS: Gamma Distribution, Mean, Variance, Reliability Theory, Moment Generating Function. 


\section{INTRODUCTION}

The Gamma distribution as a tool for probabilistic evaluation is the appropriate model for predicting random variables (r.v. 's) that are variates of the continuous type. A mathematical theory of probability is concerned with the analysis of random phenomena. The outcome of a random event cannot be determined before it occurs, but it may be any one of several outcomes and its actual outcome is considered to be determined by chance (Grimmett and stirzaker, 2001).

Conventionally, probabilities are measured or taken from the number 0 to 1 , where 0 is the minimum value and 1 is the maximum value. If random observations (objects) are obtained by repeating an experiment a very large number of times. Some fractions of this very large number of trails of an experiment will result in event A, another in B and so on. From a practical point of view, the fraction of resulting in event $\mathrm{A}$ is called the probability of $\mathrm{A}$. Consequently, if an experiment is repeated a large number of times $\mathrm{N}$ and the event $\mathrm{A}$ is observed $\mathrm{n}$ times, then the probability of A, is denoted by $p(A)=n / N$ (Hogg and Tamis, 1993). The gamma distribution arises where one is concerned about the waiting time for a finite number of independent events to occur; and the gamma distribution varies from the case of one, two, three and four parameters.

A random variable $\mathrm{X}$ is said to have the two parameter (standard) gamma distribution if its distribution is given by

$f(x)=\frac{1}{\Gamma(\alpha) \theta^{\alpha}} x^{\alpha-1} e^{\frac{-x}{\theta}}, \quad$ or $\quad f(x)=\frac{\theta^{\alpha} X^{\alpha-1} e^{-\theta x}}{f(\alpha)}, \quad x>0, \alpha>0, \theta>0$

Where the parameter $\alpha$ is called the shape parameter, since it influences the peak of the distribution (Stephenson et al., 1999). The parameter $\theta$ is called the inverse scale parameter, since most of its influence is on the spread of the distribution, i.e. the standard deviation of the gamma distribution is proportional to $\frac{1}{\theta}$. The two parameter gamma and the two parameter Weibull distribution are the most popular distributions for analyzing lifetime data (Rameshwar, 2001).

Gamma distributions are very versatile; they give useful representation of many physical situations. They are perhaps the most applied statistical distributions in the area of reliability (Saralees, 2008), and it can also be used to model the amount of daily rainfall in the region (Das, 1955; Stephenson et al., 1999). A gamma distribution was postulated because precipitation occurs only when water particles form around dust of sufficient mass, and waiting for the aspect implicit in the gamma distribution.

The purpose of this paper is to explore some of the relevant properties of the gamma distribution and its applications to real life which objectives are:

- To unveil the properties and characteristics of gamma distribution such as its mean, variance, moment generating function and its special cases.

- To highlight various applications of gamma distribution to real life situations such as fitting the gamma distribution to data, burn out-time of products and applications of gamma distribution to reliability theory. 
The rest of this paper is organized as follows: section 2 discusses the related literature. Section 3 is about material and methods with respect to gamma distribution. Furthermore, section 4 is the application and illustrative example of gamma distribution. Subsequently, section 5 concludes the paper.

\section{RELATED LITERATURE}

Some researchers seldom refer to one parameter gamma distribution as the standard gamma while others refer to the two parameter gamma as the standard gamma (Saralees. 2008). In this paper, we shall start by considering the one parameter gamma distribution case first. A random variable $X$ is said to have one parameter (standard) gamma distribution if its distribution is given by

$f(x)=\frac{X^{\alpha-1} e^{-x}}{\Gamma(\alpha)}, \quad x>0, \alpha>0$

Where the parameter $\alpha$ is called the shape parameter, since it most influences the peak of the distribution (Stephenson et al., 1999).

The two parameters of the gamma distribution like we have in Equation (1) represent the shape and scale parameters and because of the shape and scale parameters, it is quite flexible to analyze any positive real data. It has increasing and as well as decreasing failure rate depending on the shape parameter, which gives an extra edge over exponential distribution which has only constant failure rate (Gauss et al., 2012).

The most general form of gamma distribution is the three parameter generalized gamma distribution (Stacy, 1962). The distribution is suitable for modeling data with different types of hazard rate functions, decreasing, and bathtub shaped and unimodal, which makes it particularly useful for estimating individual hazard functions. The generalized gamma distribution has been used in several research areas such as engineering, hydrology and survival analysis.

The generalized gamma distribution plays a very important role in statistical inferential problems. When modeling monotone hazard rates, the Weibull distribution may be the initial choice because of its negatively and positively skewed density shapes. However, the Weibull distribution does not provide a reasonable parametric fit for modeling phenomena with nonmonotone failure rates, such as the bathtub shaped and the unimodal failure rates, which are common in biological and reliability studies (Gauss, et al., 2011). A new family of distributions generated by the gamma random variables have been recently introduced (Zografos and Balakrishnan, 2009). This family of distributions has their cumulative distribution function as $G(x)=\frac{1}{\Gamma(\alpha)} \int_{0}^{-\log \underline{F}(x)} \quad t^{\delta-1} e^{-t} d t, x \in R, \delta>$ 0

Where $\underline{F}(x)$ is the survival function which is used in generating new distributions. 


\section{METHODOLOGY}

Before discussing the gamma distribution, we will first introduce a function that is very important not only in probability theory but in many areas of mathematics which is the gamma function.

\section{Gamma Function}

The gamma function which is denoted by $\Gamma(\alpha)$ is defined as

$\Gamma(\alpha)=\int_{0}^{\infty} \quad x^{\alpha-1} e^{-x} d x, \alpha>0$

It can be shown that the above integral exist whenever $\alpha>0$ if we integrate Equation (3) by part using the formula $\int u d v=u v-\int \quad v d u$ and letting $\mathrm{u}=x^{\alpha-1}$ and $\mathrm{dv}=e^{-x}$, observe that $\mathrm{v}$ which is the integration of $\mathrm{dv}$ is $-e^{-x}$ while du which is the differentiation of $\mathrm{u}$ is $(\alpha-1) x^{\alpha-2}$. so, substituting into the $\int \quad u d v=u v-\int \quad v d u$ formula gives

$$
\begin{aligned}
& \Gamma(\alpha)=\int_{0}^{\infty} \quad x^{\alpha-1} e^{-x} d x=\left[-x^{\alpha-1} e^{-x} d\right]_{0}^{\infty}-\int_{0}^{\infty}-e^{-x}(\alpha-1) x^{\alpha-2} d x \\
& =0+\alpha-1 \int_{0}^{\infty} \quad e^{-x} x^{\alpha-2} d x=\alpha-1 \int_{0}^{\infty} \quad e^{-x} x^{\alpha-2} d x \\
& =\alpha-1\left[\left[-x^{\alpha-1} e^{-x}\right]_{0}^{\infty}-\int_{0}^{\infty}-e^{-x}(\alpha-2) x^{\alpha-3} d x\right] \\
& =\alpha-1\left[0+\alpha-2 \int_{0}^{\infty} e^{-x} x^{\alpha-3} d x\right] \\
& \therefore \Gamma(\alpha)=\alpha-1(\alpha-2) \int_{0}^{\infty} e^{-x} x^{\alpha-3} d x \ldots=(\alpha-1) \Gamma(\alpha-1)
\end{aligned}
$$

However, from Equation (3) when $\alpha=1$ gives

$$
\Gamma(1)=\int_{0}^{\infty} e^{-x} d x=\left[-e^{-x}\right]_{0}^{\infty}=\left[0-\left(-e^{-0}\right]=1\right.
$$

Thus, when $\mathrm{n}$ is a positive integer, we have that $\Gamma(n)=(n-1)$ !, For this reason; the gamma function is called the generalized factorial.

\section{Properties of Gamma Function}

For any positive real number $\alpha$ :

- $\quad \Gamma(\alpha)=\int_{0}^{\infty} x^{\alpha-1} e^{-x} d x$

This is the gamma function which is stated in Equation (3)

- $\int_{0}^{\infty} x^{\alpha-1} e^{-\lambda x} d x=\frac{\Gamma(\alpha)}{\lambda^{\alpha}}$, for $\lambda>0$; 
The above equation is also known as the gamma distribution which is mathematically restated as

$$
\int_{0}^{\infty} \quad f(x) d x=\int_{0}^{\infty} \frac{\lambda^{\alpha} x^{\alpha-1} e^{-\lambda x}}{\Gamma\left({ }^{\alpha} \alpha\right)} d x=\frac{\lambda^{\alpha}}{\Gamma\left({ }^{\prime} \alpha\right)} \int_{0}^{\infty} \quad x^{\alpha-1} e^{-\lambda x} d x
$$

Using change of variable, let $y=\lambda x, x=\frac{y}{\lambda}$, and $d x=\frac{d y}{\lambda}$ then

$$
\begin{aligned}
& \frac{\lambda^{\alpha}}{\Gamma(\alpha)} \int_{0}^{\infty} \quad x^{\alpha-1} e^{-\lambda x} d x=\frac{\lambda^{\alpha}}{\Gamma(\alpha)} \int_{0}^{\infty}\left(\frac{y}{\lambda}\right)^{\alpha-1} e^{-y} \frac{1}{\lambda} d y=\frac{\lambda^{\alpha}}{\Gamma(\alpha)} \int_{0}^{\infty} y^{\alpha-1} \frac{1}{\lambda^{\alpha-1}} e^{-y} \frac{1}{\lambda} d y \\
& =\frac{\lambda^{\alpha}}{\lambda^{\alpha-1} \Gamma(\alpha) \lambda} \int_{0}^{\infty} \quad y^{\alpha-1} e^{-y} d y=\frac{\lambda^{\alpha-\alpha+1}}{\Gamma(\alpha) \lambda} \int_{0}^{\infty} \quad y^{\alpha-1} e^{-y} d y=\frac{\lambda}{\Gamma(\alpha) \lambda} \int_{0}^{\infty} y^{\alpha-1} e^{-y} d y
\end{aligned}
$$

But $\int_{0}^{\infty} \quad y^{\alpha-1} e^{-y} d y=\Gamma(\alpha)$, hence $\int_{0}^{\infty} f(x) d x=\frac{1}{\Gamma(\alpha)} \Gamma(\alpha)=1$

$$
\text { - } \quad \Gamma(\alpha+1)=\alpha \Gamma(\alpha)
$$

Proof: $\Gamma(\alpha+1)=\int_{0}^{\infty} e^{-t} t^{\alpha} d t$

Using integration by parts; let $u=t^{\alpha}$ implies that $d u=a t^{\alpha-1} d t$ and $d v=e^{-t} d t$ which also implies that $v=-e^{-t}, \quad$ therefore $\quad \Gamma(\alpha+1)=\left[-t^{\alpha} e^{-t}\right]_{0}^{\infty}+\int_{0}^{\infty} t^{\alpha} e^{-t} d t=$ $\alpha \int_{0}^{\infty} \quad e^{-t} t^{\alpha-1} d t$

Hence $\Gamma(\alpha+1)=\alpha \Gamma(\alpha) ; \alpha>0$.

- $\quad \Gamma(n)=(n-1)$ ! for $n=1,2,3, \ldots$

It suffices from Equation (3) that when $\alpha=1$ gives

$$
\Gamma(1)=\int_{0}^{\infty} \quad e^{-x} d x=\left[-e^{-x}\right]_{0}^{\infty}=\left[0-\left(-e^{-0}\right]=\right.
$$

1

Thus, from Equation (4) when $\mathrm{n}$ is a positive integer, we have that $\Gamma(n)=(n-1)$ !, for this reason; the gamma function is called the generalized factorial.

- $\quad \Gamma\left(\frac{1}{2}\right)=\sqrt{\pi}$.

Proof: Let $\Gamma\left(\frac{1}{2}\right)=\sqrt{\pi}$, we substitute $\alpha=\frac{1}{2}$ into Equation (3) gives

$\Gamma(1 / 2)=\int_{0}^{\infty} \quad x^{1 / 2-1} e^{-x} d x=\int_{0}^{\infty} \quad x^{-1 / 2} e^{-x} d x$

If we let $x=u^{2}$, we have $d x=2 u d u$ and substituting it into the above equation gives

$\Gamma(1 / 2)=\int_{0}^{\infty} \quad u^{(2)\left(-\frac{1}{2}\right)} e^{-u^{2}} 2 u d u=\int_{0}^{\infty} \quad u^{-1} e^{-u^{2}} 2 u d u=2 \int_{0}^{\infty} \quad e^{-u^{2}} d u$.

This is called the Gaussian integral.

To derive $\Gamma(1 / 2)$, we explicitly solved for first, square the value for $\Gamma\left(\frac{1}{2}\right)$ and rewrite as a double integral, i.e. 
$\left[\Gamma\left(\frac{1}{2}\right)^{2}\right]=\left[\Gamma\left(\frac{1}{2}\right)\right]\left[\Gamma\left(\frac{1}{2}\right)\right]=\left[2 \int_{0}^{\infty} e^{-u^{2}} d u\right]\left[2 \int_{0}^{\infty} e^{-v^{2}} d v\right]$

$4 \int_{0}^{\infty} \int_{0}^{\infty} e^{-\left(u^{2}+v^{2}\right)} d v d u$

The bivariate transformation $u=r \cos \theta, v=r \sin \theta$ will transform the integral problem from Cartesian coordinates to polar coordinates, $(r, \theta)$. These new variables will range from $0 \leq r \leq \infty$ and $0 \leq \theta \leq \frac{\pi}{2}$. The Jacobian of the transformation is

$|J|=\left|\frac{\partial u}{\partial v} \frac{\partial u}{\partial \theta} \frac{\partial v}{\partial r} \frac{\partial v}{\partial \theta}\right|=|\cos \cos \theta \theta \sin \sin \theta \theta|=r \cos ^{2} \theta+r \sin ^{2} \theta=r$.

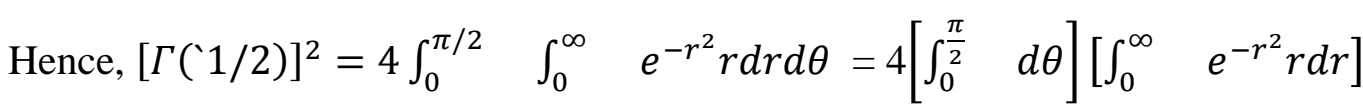

When $u=-r^{2}=>d u=-2 r d r$ and $r d r=-\frac{1}{2} d u$

$\left[\Gamma\left(\frac{1}{2}\right)\right]^{2}=4\left[\frac{\pi}{2}\right]\left[-\frac{1}{2} \int_{0}^{\infty} \quad e^{u} d u\right]=-\pi[0-1]=\pi$

$\therefore \Gamma(1 / 2)=\sqrt{\pi}$

\section{Gamma Distribution and its Characteristics}

The random variable $\mathrm{X}$ has a gamma distribution if its probability density function (p.d.f.) is defined by

$f(x)=\frac{1}{\Gamma(\alpha) \theta^{\alpha}} x^{\alpha-1} e^{\frac{-x}{\theta}}, \quad 0 \leq x<\infty$

Hence, $\mathrm{W}$, the waiting time until the $\alpha$ th change in the Poisson process, has a gamma distribution with parameters $\alpha$ and $\theta=1 / \lambda$. That is to say that when we substitute $\theta=1 / \lambda$ into equation (1) gives

$$
\begin{aligned}
& f(x)=\frac{1}{\Gamma(\alpha)\left(\frac{1}{\lambda}\right)^{\alpha}} x^{\alpha-1} e^{\frac{-x}{\left(\frac{1}{\lambda}\right)}} \\
& f(x)=\frac{1}{\Gamma(\alpha) \frac{1}{\lambda^{\alpha}}} x^{\alpha-1} e^{-\lambda x}
\end{aligned}
$$

$\infty$

$$
f(x)=\frac{\lambda^{\alpha} x^{\alpha-1} e^{-\lambda x}}{\Gamma(\alpha)}, 0 \leq x<
$$

Which is another form of the gamma distribution, and an alternative form of the gamma distribution is given $f(x)=\frac{\lambda(\lambda x)^{\alpha-1} e^{-\lambda x}}{\Gamma(\alpha)}, 0 \leq x<$ $\infty$

\section{Mean of the Gamma Distribution}

The Mean of $\mathrm{x}$ (or the mean of the distribution) which is $\mathrm{E}(\mathrm{x})$ is denoted by $\mu=E(x)$, called the first moment. So, the $\mathrm{E}(\mathrm{x})$ for the continuous case of the random variables is given by 


$$
\begin{aligned}
& E(x)= \\
& \int_{0}^{\infty} x f(x) d x
\end{aligned}
$$

But $f(x)=\frac{\lambda}{\Gamma(\alpha)}(\lambda x)^{\alpha-1} e^{-\lambda x}$

$E(x)=\int_{0}^{\infty} \frac{x \lambda}{\Gamma(\alpha)}(\lambda x)^{\alpha-1} e^{-\lambda x} d x=\int_{0}^{\infty} \frac{(\lambda x)^{\alpha}}{\Gamma(\alpha)} e^{-\lambda x} d x=\frac{\lambda^{\alpha}}{\Gamma(\alpha)} \int_{0}^{\infty} \quad x^{\alpha} e^{-\lambda x} d x$

By change of variable, let $y=\lambda x, x=\frac{y}{\lambda}$ and $d x=\frac{d y}{\lambda}$

$$
\begin{aligned}
& E(x)=\frac{\lambda^{\alpha}}{\Gamma(\alpha)} \int_{0}^{\infty}\left(\frac{y}{\lambda}\right)^{\alpha} e^{-y} \frac{d y}{\lambda}=\frac{\lambda^{\alpha}}{\Gamma(\alpha)} \int_{0}^{\infty} y^{\alpha} \frac{1}{\lambda^{\alpha+1}} e^{-y} \frac{d y}{\lambda}=\frac{\lambda^{\alpha}}{\Gamma(\alpha)} \frac{1}{\lambda^{\alpha+1}} \int_{0}^{\infty} y^{\alpha} e^{-y} d y \\
& E(x)=\frac{\lambda^{\alpha}}{\Gamma(\alpha)} \frac{1}{\lambda^{\alpha+1}} \Gamma(\alpha+1)
\end{aligned}
$$

Recall that by recurrence formula of the gamma function

$\Gamma(\alpha+1)=\alpha \Gamma(\alpha)$. This implies that $E(x)=\frac{\lambda^{\alpha} \alpha \Gamma(\alpha)}{\Gamma(\alpha) \lambda^{\alpha} \lambda}$

$\therefore E(x)=\frac{\alpha}{\lambda}$. This is the mean of the gamma distribution with parameter $\lambda$ and $\alpha$.

\section{Variance of the Gamma Distribution}

The variance of a distribution is denoted by

$\operatorname{Var}(x)=E\left(x^{2}\right)-[E(x)]^{2}$

To obtain the variance of the gamma distribution, we first obtain the first moment, then the second moment which is denoted by $E\left(x^{2}\right)$.

$$
\begin{aligned}
& E\left(x^{2}\right)= \\
& \int_{0}^{\infty} x^{2} f(x) d x
\end{aligned}
$$

Where $f(x)$ is the probability density function (p.d.f.), which is equal to $\frac{\lambda(\lambda x)^{\alpha-1} e^{-\lambda x}}{\Gamma(\alpha)}$

$E\left(x^{2}\right)=\int_{0}^{\infty} \frac{x^{2} \lambda(\lambda x)^{\alpha-1} e^{-\lambda x}}{\Gamma(\alpha)} d x=\int_{0}^{\infty} \frac{x^{2} \lambda^{\alpha} x^{\alpha-1} e^{-\lambda x}}{\Gamma(\alpha)} d x=\int_{0}^{\infty} \frac{\lambda^{\alpha} x^{\alpha+1} e^{-\lambda x}}{\Gamma(\alpha)} d x=$ $\frac{\lambda^{\alpha}}{\Gamma(\alpha)} \int_{0}^{\infty} x^{\alpha+1} e^{-\lambda x} d x$

By change of variable, let $y=\lambda x, x=\frac{y}{\lambda}, d x=\frac{d y}{\lambda}$ then

$E\left(x^{2}\right)=\frac{\lambda^{\alpha}}{\Gamma(\alpha)} \int_{0}^{\infty} \quad\left(\frac{y}{\lambda}\right)^{\alpha+1} e^{-y} \frac{1}{\lambda} d y=\frac{\lambda^{\alpha}}{\Gamma(\alpha)} \int_{0}^{\infty} \frac{y^{\alpha+1} e^{-y}}{\lambda^{\alpha+2}} d y=\frac{\lambda^{\alpha}}{\Gamma(\alpha) \lambda^{\alpha+2}} \int_{0}^{\infty} \quad y^{\alpha+1} e^{-y} d y$ $=\frac{\lambda^{\alpha}}{\Gamma(\alpha) \lambda^{\alpha+2}} \Gamma(\alpha+2)$. By recurrence of the gamma function, $\Gamma(\alpha+2)=\alpha(\alpha+1) \Gamma(\alpha)$

$E\left(x^{2}\right)=\frac{\lambda^{\alpha} \alpha(\alpha+1) \Gamma(\alpha)}{\Gamma(\alpha) \lambda^{\alpha+2}}=\frac{\lambda^{\alpha} \alpha(\alpha+1) \Gamma(\alpha)}{\Gamma(\alpha) \lambda^{\alpha} \lambda^{2}}=\frac{\alpha(\alpha+1)}{\lambda^{2}}$ 


$$
\begin{aligned}
& \operatorname{Var}(x)=E\left(x^{2}\right)-[E(x)]^{2} \\
& \operatorname{Var}(x)=\frac{\alpha(\alpha+1)}{\lambda^{2}}-\frac{\alpha^{2}}{\lambda^{2}}=\frac{\alpha^{2}+\alpha-\alpha^{2}}{\lambda^{2}} \\
& \therefore \operatorname{Var}(x)=\frac{\alpha}{\lambda^{2}}
\end{aligned}
$$

\section{Moment Generating Function of Gamma Distribution}

By definition: $M_{x}(t)=E\left(e^{t x}\right)=\int_{0}^{\infty} e^{t x} f(x) d x$

Given that the p.d.f. of gamma distribution is $F(x)=\frac{\lambda(\lambda x)^{\alpha-1} e^{-\lambda x}}{\Gamma(\alpha)}$

Then $\quad M \quad{ }_{x}(t)=\int_{0}^{\infty} \frac{e^{t x} \lambda(\lambda x)^{\alpha-1} e^{-\lambda x}}{\Gamma(\alpha)} d x=\int_{0}^{\infty} \frac{e^{t x} \lambda^{\alpha} x^{\alpha-1} e^{-\lambda x}}{\Gamma(\alpha)} d x=$ $\frac{\lambda^{\alpha}}{\Gamma(\alpha)} \int_{0}^{\infty} x^{\alpha-1} e^{-\lambda x+t x} d x$

$=\frac{\lambda^{\alpha}}{\Gamma(\alpha)} \int_{0}^{\infty} \quad x^{\alpha-1} e^{-x(\lambda-t)} d x$. By change of variable, let $y=x(\lambda-t), x=\frac{y}{(\lambda-t)}$ then $d x=$ $\frac{d y}{(\lambda-t)}$

$M_{x}(t)=\frac{\lambda^{\alpha}}{\Gamma(\alpha)} \int_{0}^{\infty} \quad\left(\frac{y}{\lambda-t}\right)^{\alpha-1} e^{-x(\lambda-t)} \frac{d y}{(\lambda-t)}=\frac{\lambda^{\alpha}}{\Gamma(\alpha)} \int_{0}^{\infty} \quad y^{\alpha-1}\left(\frac{y}{\lambda-t}\right)^{\alpha-1+1} e^{-y} d y$

$M \quad x(t)=\frac{\lambda^{\alpha}}{\Gamma(\alpha)} \int_{0}^{\infty} \quad y^{\alpha-1} \frac{1}{(\lambda-t)^{\alpha}} e^{-y} d y=\frac{\lambda^{\alpha}}{(\lambda-t)^{\alpha}} \frac{1}{\Gamma(\alpha)} \int_{0}^{\infty} \quad y^{\alpha-1} e^{-y} d y$

Recall that $\int_{0}^{\infty} y^{\alpha-1} e^{-y} d y=\Gamma(\alpha)$

Then $M \quad{ }_{x}(t)=\frac{\lambda^{\alpha}}{(\lambda-t)^{\alpha}} \frac{\Gamma(\alpha)}{\Gamma(\alpha)} \therefore M \quad{ }_{x}(t)=\frac{\lambda^{\alpha}}{(\lambda-t)^{\alpha}}=\left(\frac{\lambda}{\lambda-t}\right)^{\alpha}$

Which is the moment generating function of the gamma distribution with parameter $\lambda$ and $\alpha$.

\section{Some Special Case of Gamma Distribution}

The gamma distribution has some special cases which include the chi square distribution, exponential distribution and the Erlang distribution.

\section{Chi-Square Distribution}

The gamma distribution with parameters $\lambda$ and $\alpha$ has a p.d.f. given by

$f(x)=\frac{\lambda(\lambda x)^{\alpha-1} e^{-\lambda x}}{\Gamma(\alpha)}=\frac{\lambda^{\alpha} x^{\alpha-1} e^{-\lambda x}}{\Gamma(\alpha)} ;$ let $\lambda=\frac{1}{2}$ and $\alpha=\frac{r}{2}$ Where $r$ is a positive integer, then the p.d.f. of $\mathrm{x}$ is called the chi-square distribution given as

$$
f(x)=\frac{1}{\Gamma\left(\frac{r}{2}\right)^{\frac{r}{2}}} x^{\frac{r}{2}-1} e^{-\frac{x}{2}}, \quad 0, \leq x<
$$

$\infty$ 
With $\mathrm{r}$ degree of freedom, which we abbreviate by saying, $x$ is $x_{(r)}^{2}$, the moment generating function of the chi-square distribution is given as:

$$
M \quad{ }_{x}(t)=\frac{1}{(1-2 t)^{\frac{r}{2}}}=(1-2 t)^{-\frac{r}{2}}, \text { which mean } E(x)=r \text { and variance } \operatorname{var}(x)=2 r .
$$

\section{Exponential Distribution}

The gamma distribution has parameter $\lambda$ and $\alpha$, when $\alpha=1$, the result is the exponential distribution with parameter $\lambda$. The exponential distribution with parameter $\lambda$ is given by

$$
\begin{aligned}
& f(x)=\left\{\begin{array}{l}
\lambda e^{-\lambda x}, \quad x \\
>00, \text { elsewhere }
\end{array}\right.
\end{aligned}
$$

Hence, the moment generating function of the exponential distribution is given by

$M_{x}(t)=\frac{\lambda}{\lambda-t}$. Thus, $E(x)=\frac{1}{\lambda}$ and $\operatorname{var}(x)=1 / \lambda^{2}$.

\section{Erlang Distribution}

The erlang distribution is a special case of the gamma distribution with parameters $\lambda$ and $\alpha$, when $\alpha=k$ and also recall that $\Gamma(k)=(k-1)$ !, then the p.d.f. of the erlang distribution becomes

$$
f(x)=\frac{\lambda^{k} x^{k-1}}{(k-1) !} e^{-\lambda x}, \quad x>0
$$

The moment generating function is given as $M_{x}(t)=\left(1-\frac{t}{\lambda}\right)^{-k}$ for $t<\lambda$

Thus, $E(x)=\frac{k}{\lambda}$ and $\operatorname{var}(x)=\frac{k}{\lambda^{2}}$

\section{Application of Gamma Distribution}

The Gamma distribution and its application is extremely important in risk analysis modeling, with a number of different uses like:

- Reliability and queuing theory: Examples include the distribution of failure times of components, the distribution of times between calibration of instruments which need re-calibration after a certain number of uses and the distribution of waiting times of $\mathrm{k}$ customers who will arrive at a store.

- Poisson waiting time: The Gamma $(\alpha, \theta)$ distribution models the time required for $\alpha$ events to occur, given that the events occur randomly in a Poisson process with a mean time between events of $\theta$. For example, if we know that major flooding occurs in a town on average every six years, Gamma $(4,6)$ models how many years it will take before the next four floods occur.

- Hydrological Analysis: In hydrology, the gamma distribution has the advantage of having only positive values, since hydrological variables such as rainfall and runoff are always positive (greater than zero) or equal to zero as a lower limit value (Markovic., 1965). 


\section{Fitting the Gamma Distribution to Data}

Given any set of data, we first show how we can determine the parameter $\lambda$ and $\propto$ before fitting it to the given data. Recall that $E(x)=\propto / \lambda$ and $\operatorname{var}(x)=\frac{\alpha}{\lambda^{2}}$, are respectively mean and variance of the gamma distribution. To find $\lambda$, using the expression for the mean, we have

$\propto=\lambda E(x)$

And from that of the $\operatorname{var}(x)$, we get

$\propto=\lambda^{2} \operatorname{var}(x)$

Equating (13) and (14), we have

$\lambda E(x)=\lambda^{2} \operatorname{var}(x)$ implies that $E(x)=\lambda \operatorname{var}(x)$, then $\lambda=\frac{E(x)}{\operatorname{var}(x)}$ where $E(x)=\frac{\alpha}{\lambda}$ hence

$\lambda=$

$\frac{\propto}{\lambda \operatorname{var}(x)}$

To find the parameter $\propto$, from Equation (13) , we have

$\lambda=$
$\frac{\alpha}{E(x)}$

From Equation (14), we have

$\lambda^{2}=\frac{\propto}{\operatorname{var}(x)}$

$\lambda=$

$\sqrt{\frac{\alpha}{\operatorname{var}(x)}}$

Combining Equating (16) and (17), we have

$\frac{\propto}{E(x)}=\sqrt{\frac{\alpha}{\operatorname{var}(x)}}$ implies that $\frac{\alpha^{2}}{[E(x)]^{2}}=\frac{\propto}{\operatorname{var}(x)}$ which also implies that $\propto^{2} \operatorname{var}(x)=\propto[E(x)]^{2}$

$\propto \operatorname{var}(x)=[E(x)]^{2}$

$\propto=$

$\frac{[E(x)]^{2}}{\operatorname{var}(x)}$

Hence, given any set of data to obtain the parameter $\lambda$ and $\propto$ of the gamma distribution, we first compute the mean $E(x)$ and then the variance $\operatorname{var}(x)$ of the data and afterwards apply accordingly. 


\section{Illustrative Example 1}

The raw data used in the example is obtained from the day to day log-book of the technical department of Niger-Calabar Flour Mill at Cross River State Nigeria.

Table 1: Raw Failure Data

\begin{tabular}{|c|l|l|l|l|l|l|l|l|l|}
\hline Observed failures $i$ & 1 & 2 & 3 & 4 & 5 & 6 & 7 & 8 & 9 \\
\hline Failure times in years, $x_{i}$ & 0.41 & 0.58 & 0.75 & 0.85 & 1.00 & 1.08 & 1.17 & 1.25 & 1.35 \\
\hline
\end{tabular}

The ordered failure times were observed failure times of a piston right in the milling machine from 2018-2019. The failure was due to "oil shortage". Using the gamma distribution, we approximate the distribution of failure times. To find the value $\lambda$ and $\propto$ from the data, we first find the mean, $E(x)$ and variance, $\operatorname{var}(x)$ from the above data.

\begin{tabular}{|l|l|l|}
\hline.$X_{i}$ & $X_{i}-\underline{x}$ & $\left(X_{i}-\underline{x}\right)^{2}$ \\
\hline 0.41 & -0.46 & 0.2116 \\
0.58 & -0.29 & 0.0841 \\
0.75 & -0.72 & 0.5184 \\
0.83 & -0.04 & 0.0016 \\
1.00 & 0.13 & 0.0169 \\
1.08 & 0.21 & 0.0441 \\
1.17 & 0.30 & 0.0900 \\
1.25 & 0.38 & 0.1444 \\
1.35 & 0.48 & 0.2304 \\
& & \\
\hline$\sum_{i=1}^{n} x_{i}=7.82$ & & $\sum_{i=1}^{n}\left(X_{i}-\underline{x}\right)^{2}=1.3415$ \\
\hline
\end{tabular}

$E(x)=\sum_{i=1}^{n} \quad x_{i}=\frac{1}{9}[0.41+0.58+\cdots+1.35]=\frac{1}{9}[7.82]=0.87$

$\operatorname{Var}(x)=\frac{1}{n} \sum_{i=1}^{n} \quad\left(X_{i}-\underline{x}\right)^{2}=\frac{1}{9}[1.3415]=0.149$. Recall that $\lambda=\frac{E(x)}{\operatorname{var}(x)}=\frac{0.87}{0.149}=$ 5.839 .

And $\propto=\frac{[E(x)]^{2}}{\operatorname{var}(x)}=\frac{(0.87)^{2}}{0.149}=5.080$

Therefore, the gamma distribution of failure times is given by

$$
\begin{aligned}
& f(x)=\frac{\lambda(\lambda x)^{\alpha-1} e^{-\lambda x}}{\Gamma(\alpha)}, x>0 \\
& f(x)=\frac{5.839(5.839 x)^{5.080-1} e^{-5.839 x}}{\Gamma(5.080)}=\frac{5.839(5.839 x)^{4.080} e^{-5.839 x}}{\Gamma(5.080)}, \mathrm{x}>0
\end{aligned}
$$




\section{Illustrative Example 2}

The burn out time of most products especially electrical devices usually has the gamma distribution.

To illustrate the application of gamma distribution to the burn out time of products, we shall take a sample of your light bulbs with time at 3.9, 4.1, 4.5, and 5.0 days.

We shall estimate the parameters using the method of moments of the above gamma distribution.

$f(x)=\frac{\lambda(\lambda x)^{\alpha-1} e^{-\lambda x}}{\Gamma(\alpha)}, x>0$

Hence, we shall obtain the mean $E(x)$ and $\operatorname{var}(x)$. Furthermore, we shall make two assumptions:

$\mu_{1}=\frac{\propto}{\lambda}=\frac{1}{n} \sum_{i=1}^{n} \quad x_{i}$

$\mu_{2}=\delta^{2}(x)+\left(\mu_{1}\right)^{2}=\frac{\alpha}{\lambda^{2}}+\left(\frac{\alpha}{\lambda}\right)^{2}$

$\mu_{1}=\frac{\propto}{\lambda^{2}(\alpha+1)}=\frac{1}{n} \sum_{i=1}^{n} \quad x_{i}^{2}$

It is true that the moment generating function of the gamma ray distribution is

$M_{x}(t)=\left[\frac{\lambda}{\lambda-t}\right]^{\propto}$. By our assumption, $\mu_{1}=\frac{1}{n} \sum_{i=1}^{n} \quad x_{i}=\frac{1}{4}(3.9+4.1+4.5+5.0)=\frac{17.5}{4}=$ 4.375

$\mu_{2}=\frac{1}{n} \sum_{i=1}^{n} \quad x_{i}^{2}=\frac{1}{4}\left((3.9)^{2}+(4.1)^{2}+(4.5)^{2}+(5.0)^{2}\right)=\frac{1}{4}[15.21+16.81+20.25+$ 25.0]

$\mu_{2}=\frac{1}{4}(77.27)=19.3175$

Now to find $\lambda$ and $\propto$, we solve the equations,

$\mu_{1}=E(x)=\frac{\alpha}{\lambda}=\frac{1}{n} \sum_{i=1}^{n} \quad x_{i}$

$\therefore \frac{\propto}{\lambda}=4$

$\mu_{2}=\delta^{2}(x)+\mu^{2}=\frac{\alpha}{\lambda^{2}}+\left(\frac{\propto}{\lambda}\right)^{2}=\frac{1}{n} \sum_{i=1}^{n} \quad x_{i}^{2}$

$\therefore \frac{\propto}{\lambda^{2}}+\left(\frac{\propto}{\lambda}\right)^{2}=19.3175$

From Equation (19), we have

$\propto=4.375 \lambda$ 
Substituting for $\propto$ into Equation (20) gives

$\frac{4.375 \lambda}{\lambda^{2}}+\frac{(4.375 \lambda)^{2}}{\lambda^{2}}=19.3175$

$\frac{4.375 \lambda+19.1406 \lambda^{2}}{\lambda^{2}}=19.3175$

$4.375 \lambda=19.3175 \lambda^{2}-19.1406 \lambda^{2}$

$4.375 \lambda=0.1769 \lambda^{2}$

$\lambda=\frac{4.375}{0.1769}=24.7315$

Put Equation (21) in (19) to obtain $\propto$ i.e.

$\frac{\propto}{24.7315}=4$

$\therefore \propto=4 \times 24.7315=98.926$

Hence, fitting this to the gamma distribution gives

$f(x)=\frac{\lambda(\lambda x)^{\alpha-1} e^{-\lambda x}}{\Gamma(\alpha)}=\frac{24.7315(24.7315 x)^{98.926-1} e^{-24.7315 x}}{\Gamma(98.926)}=\frac{24.7315(24.7315 x)^{97.926} e^{-24.7315 x}}{\Gamma(98.926)}$

Which is the probability density function for the burn out time $\mathrm{x}$ of the bulbs (light bulbs).

\section{CONCLUSION}

In this paper, we have presented the study of properties and applications of gamma distribution to real life situations such as fitting the gamma distribution into data, burn-out time of electrical devices and reliability theory. It was also shown that gamma distribution is a legitimate continuous probability distribution with moment generating function $\left(M_{x} t\right)=$ $\left(\frac{\lambda}{\lambda-t}\right)^{\alpha}, \quad E(x)=\frac{\alpha}{\lambda}$ and $\operatorname{var}(x)=\frac{\alpha}{\lambda^{2}}$.

\section{REFERENCES}

Das, S. C. (1955), Fitting Truncated Type III Curves to Rainfall Data. Australian Journal of Physics, 8, 298-304.

Gauss M., Fredy, C., Lourdes C., Montenegro (2012). The beta generalized gamma distribution, Statistics: A Journal of Theoretical and Applied Statistics, DOI:10.1080/02331888.2012.658397

Gauss M., Edwin M., Ortega M and Giovana, O. (2011). The exponentiated Generalized gamma distribution with application to lifetime data, Journal of Statistical Computation and Simulation, 81:7, 827-842. 
Grimett, G. R. and Stirzaker, D. R. (2001). Probability and Random Processes, Third Edition, Oxford University Press.

Hogg, V. R. and Tanis, A. E. (1993). Probability and Statistical Inference, Fourth Edition Macmillan Publishing Company, New York.

Markovic, R.D. (1965). Probability Functions of Best Fit to Distributions of Annual Precipitation and Runo, Hydrology Paper, No.8, Colorado State University, Fort Collins, Colorado.

Rameshwar, D. (2001). Exponentiated Exponential Family: An Alternative to Gamma and Weibull Distributions. Biometrical Journal $43,117-130$.

Saralees Nadarajah (2008). Some gamma distributions, Statistics: A Journal of Theoretical and Applied Statistics, 42:1, 77-94.

Stacy, E. W. (1962). A generalization of the gamma distribution. Ann. Math. Statist. 33, 3 (Sep), 1187-1192.

Stephenson, D. B., Kumar, K. R., Doblas-Reyes, F. J., Chauvin, E. and Pezzulli, S. (1999). Extreme daily rainfall events and their impact on Ensemble Forecast of the Indian Moonsoon. Monthly Weather Review, 127, 1954-1966.

Zografos, K. and Balakrishnan, N. (2009), On families of beta- and generalized gammagenerated distributions and associated inference. Stat. Methodol. 6 (2009), pp. 344-362. 